\title{
Die Rente als Bilanz des Lebens - Alterssicherung und Arbeit von Frauen auf dem Prüfstand
}

Jutta Schmitz-Kießler

\section{Einleitung}

Die Diskussion um die Alterseinkünfte von Frauen hält seit Jahrzehnten an: Immer wieder wird in der politischen und mehr noch der wissenschaftlichen Auseinandersetzung der empirische belastbare Befund diskutiert, dass die Frauenrenten erheblich vom Ruhestandseinkommen der Männer abweichen. In der jüngeren Debatte hat das Bundesfamilienministerium mit dem so genannten Gender Pension Gap (GPG) einen Indikator verwendet, der diese Ungleichheit auf den Punkt bringen soll. Die Maßzahl beschreibt das Verhältnis der durchschnittlichen persönlichen eigenen Alterssicherungsleistung von Frauen zu der von Männern (Flory 2011, S. 7). Dabei werden sowohl die Einkünfte aus dem gesetzlichen System als auch Betriebs- und Privatrenten in die Berechnung mit einbezogen. Außerdem wird das GPG in der Regel getrennt nach Ost- und Westdeutschland berechnet, weil beide Regionen extreme Unterschiede aufweisen: Aktuell lässt sich das GPG in Westdeutschland auf 48,8\% und in Ostdeutschland auf 20,1 \% beziffern (Hammerschmid/Rowold 2019, S. 441). Weiterführende Analysen belegen außerdem, dass die Rentenlücke bei verheirateten und verwitweten Frauen, Rentnerinnen mit niedrigem beruflichen Bildungsabschluss, einer höheren Anzahl von Kindern sowie hochaltrigen Frauen besonders groß ausfällt (Sopp/Wagner 2011).

Darüber hinaus lässt sich belegen, dass die Ursachen für das GPG in erster Linie mit der Arbeitsmarktintegration zusammenhängen (Hammerschmid/Rowold 2019, S. 441). Das ist problematisch, weil es sowohl bei der Lohnarbeit als auch der unbezahlten Sorgearbeit nach wie vor gravierende Unterschiede zwischen Männern und Frauen gibt. Außerdem haben die Sozialreformen der letzten zwanzig Jahre in Deutschland dazu geführt, dass die Alterseinkünfte nicht nur immer deutlicher von der vorherigen Erwerbsbiografie abhängen, sondern die Arbeitsmarktrisiken in zunehmendem Maße individualisiert werden und voll auf die Rente durchschla- 
gen (siehe den Beitrag von Czepek in diesem Band). Es liegt auf der Hand, dass es nach wie vor insbesondere Frauen sind, die Gefahr laufen, nur niedrige (eigene) Alterseinkünfte zu erhalten.

Aber gibt es nicht auch Anzeichen für eine Problementschärfung? Schließlich hat sich die Frauenerwerbsbeteiligung in den letzten Jahren stetig erhöht. Auch die vertikale und horizontale Ungleichheit der Geschlechter ist - beispielsweise im Rahmen der Frauenquote - politisch behandelt worden. Lassen sich also Fortschritte verzeichnen, so dass perspektivisch damit zu rechnen ist, dass sich die Arbeitsmarktsituation von Frauen nachhaltig entspannt und damit auch ihre Möglichkeiten zur eigenständigen Altersvorsorge deutlich gestärkt wird?

Um diese Frage zu beantworten, wird die Situation von Frauen in der Rente und auf dem Arbeitsmarkt im Folgenden nachgezeichnet. Nach einer überblicksartigen Skizze der weiblichen Renteneinkommen werden die wesentliche Merkmale beschrieben, die die Arbeitsmarktsituation von Frauen kennzeichnen. In der abschließenden Diskussion wird dann argumentiert, dass neben den notwendigen Arbeitsmarktreformen auch (renten-)rechtliche Neuerungen unabdingbar sind, die gezielt die Lebenslagen von Frauen verbessern.

\section{Bestandsaufnabme: Frauenrenten}

Um die ökonomische Situation von Frauen im Alter zu skizzieren ist es notwendig, ihre Renteneinkommen aus verschiedenen Perspektiven zu betrachten. Dementsprechend werden im Folgenden die Höhe der gesetzlichen Renten sowie die Situation in der betrieblichen und privaten Altersvorsorge dargestellt. Es sei allerdings vorangestellt, dass ein Blick auf individuelle Renten immer unvollständig ist und nicht zwangsweise die tatsächliche materielle Lebenslage widerspiegelt. Schließlich können niedrige Versichertenrenten von Frauen im Haushaltskontext durch hohe Renten eines Mannes oder Partners kompensiert werden. Insofern sind die individuellen Renten allein noch kein ausreichender Indikator für eine angespannte finanzielle Lage oder Altersarmut. Dennoch ist der Blick auf die persönlichen Rentenzahlungen wichtig. Denn nur so lässt sich das Problem verdeutlichen, dass ein Großteil der Frauen nach wie vor von den Einkünften des (Ehe-)Mannes abhängig ist. Zur Bestandsaufnahme wird deswegen im Folgenden zunächst zwischen Versicherten- und Witwenrenten unterschieden, bevor die Situation von Frauen in den Systemen der betrieblichen und privaten Vorsorge umrissen wird. 


\subsection{Versichertenrenten der gesetzlichen Rentenversicherung}

Die gesetzlichen Renten werden vor allem durch die frühere Position der Versicherten auf dem Arbeitsmarkt bestimmt (siehe Abschnitt 3). Aus diesem Grund weichen Frauenrenten nicht nur stark von den Renten der Männer $\mathrm{ab}$ - die individuellen Rentenzahlungen variieren auch sowohl unter den Männern als auch unter den Frauen je nach Rentenart beträchtlich. Dementsprechend wird die ganze Spannweite von Frauen und Männerrenten nur sichtbar, wenn nicht eine (männliche bzw. weibliche) Durchschnittsrente betrachtet wird, sondern neben dem Geschlecht auch nach Rentenarten ${ }^{1}$ unterschieden wird. Außerdem müssen die Bestandsrenten (also einer Betrachtung aller Rentnerinnen und Rentner) von dem jeweiligen Beobachtungsjahr neu zugegangenen Renten abgrenzt werden. Während die Bestandsrenten die Situation von allen Männern und Frauen darstellen, können die Zugangsrenten als Trendbarometer herangezogen werden, um kürzer zurückliegende Arbeitsmarktentwicklungen zu beobachten. Schließlich geht in die Analyse der Bestandsrenten auch das schon sehr lange zurückliegende Erwerbsverhalten - und die ebenfalls teilweise sehr lang zurückliegenden Rollenmuster und geschlechtsspezifischen Verhaltensweisen - mit ein, während den Zugangsrenten aktuellere Erwerbsbiografien zugrunde liegen.

In Abbildung 1 sind zunächst die monatlich gezahlten Bestandsrenten von Männern und Frauen sowohl für Ost- als auch für Westdeutschland dargestellt. Insgesamt liegen die Altersrenten der Männer im Jahr 2018 bei $1.130 €$ (West) bzw. $1.226 €$ (Ost) und die der Frauen bei $647 €$ (West) bzw. $962 €$ (Ost). Überblicksartig lässt sich außerdem festhalten, dass

- die durchschnittlichen Renten von Frauen in allen Rentenarten (mit Ausnahme der Renten wegen verminderter Erwerbsfähigkeit in Ostdeutschland) geringer ausfallen als die Renten der Männer. Dahinter steht, dass das Erwerbsverhalten von Frauen und Männern nach wie vor stark voneinander abweicht (siehe Abschnitt 3).

- die geschlechtsspezifischen Unterschiede in Ostdeutschland deutlich geringer sind als im Westen Deutschlands. Das lässt sich darauf zurückführen, dass Frauen in der ehemaligen DDR deutlich besser in den Ar-

1 Die Rentenart ist ein wesentlicher Faktor für die Höhe der Rente, da ein Bezug vorgezogener Renten beispielsweise nur möglich ist, wenn eine lange Versicherungsdauer vorliegt. Insofern ist die durchschnittliche Höhe dieser Renten immer höher als der durchschnittliche Zahlbetrag einer Regelaltersrente, deren Bezug lediglich fünf Versicherungsjahre voraussetzt. 
beitsmarkt integriert waren und zum Teil auch heute noch sind. Die Renten der Männer weichen in den beiden Landesteilen nicht derart gravierend voneinander $\mathrm{ab}$.

- die höchsten monatlichen Zahlbeträge im Bereich der Renten für besonders langjährig Versicherte vorzufinden sind. Das ist nicht verwunderlich, weil die Voraussetzung für den Bezug dieser im Jahr 2012 eingeführten Rentenart darin besteht, 45 Pflichtbeitragsjahre vorzuweisen. Auch wenn hierzu auch Pflichtbeiträge aus Kindererziehung, Pflege, Krankengeldbezug oder Wehr- und Zivildienst zählen, können diese Rentnerinnen und Rentner in der Regel auf besonders langjährige Beschäftigungen zurückblicken. Der Zeitfaktor wirkt sich positiv auf die Höhe der Renten aus.

\section{Abbildung 1: Durchschnittliche Rentenhöhen im Rentenbestand nach Renten-} art und Geschlecht 2018, Euro

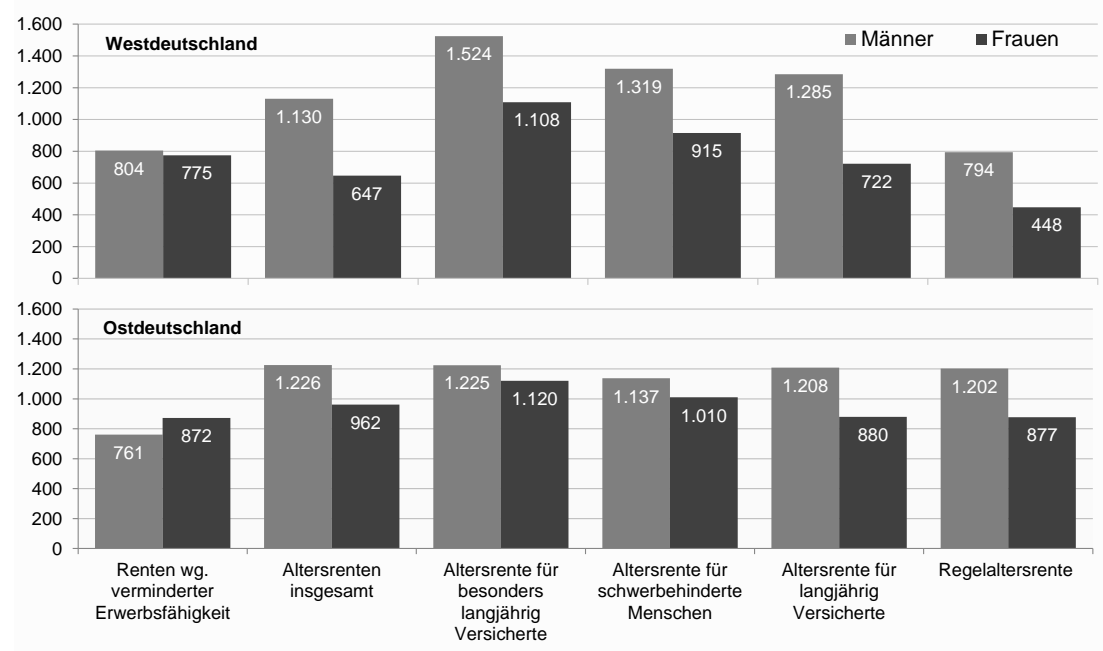

Quelle: Deutsche Rentenversicherung Bund (2019), Rentenversicherung in Zahlen, eigene Darstellung

Bei der Interpretation muss berücksichtigt werden, dass die Zugangsvoraussetzung für die Regelaltersrente lediglich darin besteht, eine Wartezeit von fünf Jahren erfüllt zu haben. Diese vergleichsweise niedrige Zugangshürde wird beispielsweise auch von Menschen erfüllt, die nur für kurze Zeit (zum Beispiel zu Beginn ihrer Erwerbstätigkeit, vor einem Wechsel in eine selbstständige Tätigkeit oder in ein Beamtenverhältnis) in der gesetzli- 
chen Rentenversicherung Beiträge gezahlt haben. Auch bei den Frauen kann diese Rentenart von denen in Anspruch genommen werden, die ihre Erwerbstätigkeit schon früh aufgeben haben oder aus anderen Gründen nur wenige Versicherungsjahre aufweisen können. Dementsprechend fallen die Rentenzahlungen hier besonders gering aus.

Die neu zugegangen Renten weisen eklatante Unterschiede zu den laufenden Bestandsrenten insgesamt auf (siehe Abbildung 2). Sie stehen zeitlich wesentlich stärker in Verbindung zu den aktuellen Verhältnissen auf dem Arbeitsmarkt und lassen erkennen, welche Folgen die neueren Entwicklungen (wie Arbeitslosigkeit, prekäre Beschäftigungsverhältnisse, Niedriglöhne) auf die Rentenhöhe haben. Das gilt sowohl für die alten als auch die neuen Bundesländer. Außerdem spiegeln sich in den Unterschieden rentenrechtliche Änderungen wider. Darüber hinaus wird sichtbar, dass die Struktur der Durchschnittsrenten im Zugang nicht grundsätzlich von der der Bestandsrenten abweicht. Daraus lässt sich schlussfolgern, dass die Entwicklung der Frauenerwerbsintegration in den vergangenen Jahrzehnten nicht stark genug ausgeprägt war, um die geschlechtsspezifische Rentenlücke sukzessive zu schließen. Stichpunktartig lässt sich festhalten, dass

- die Zahlbeträge der durchschnittlichen Zugangsrenten von Frauen in allen Rentenarten unter denen der Männer liegen (mit Ausnahme der Renten wegen verminderter Erwerbsfähigkeit in den neuen Ländern);

- die geschlechtsspezifischen Unterschiede in Ostdeutschland aber geringer ausfallen als im Westen Deutschlands;

- die Zugangsvoraussetzung langer Versichertenzeiten zu durchschnittlich höheren Renten führt, aber

- unter den Altersrenten für besonders langjährig Versicherte kein nennenswerter Unterschied zwischen dem Rentenbestand und dem Rentenzugang besteht. Das liegt aber auch daran, dass diese Rentenart erst im Jahr 2012 neu eingeführt wurde. 


\section{Abbildung 2: Durchschnittliche Rentenhöhen im Rentenzugang nach Rentenart und Geschlecht 2018, Euro}

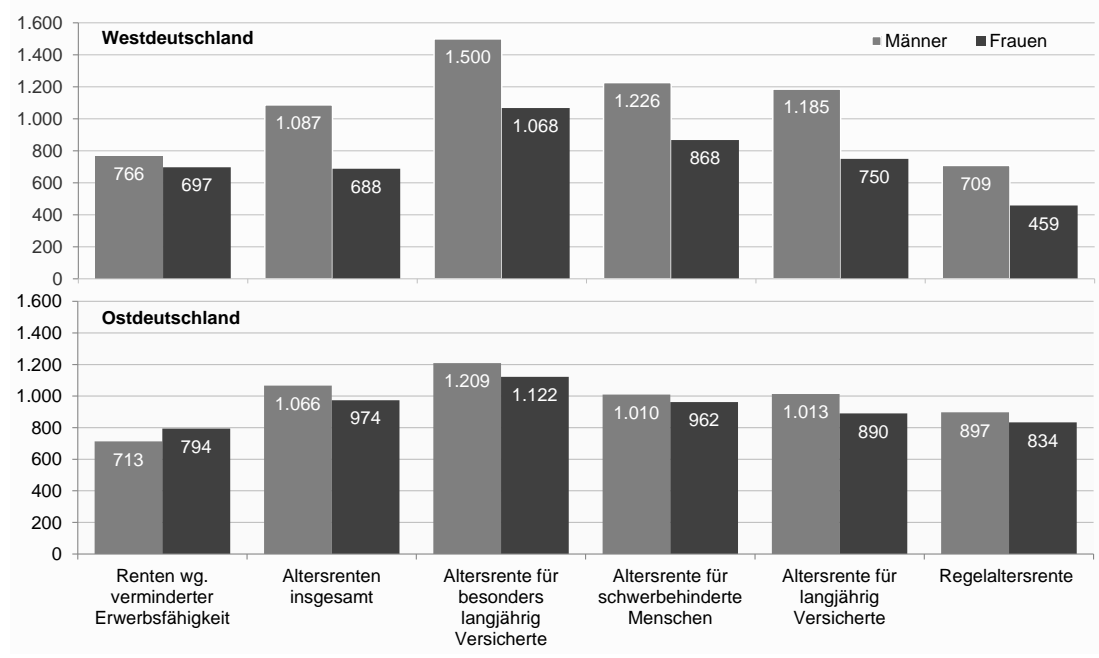

Quelle: Deutsche Rentenversicherung Bund (2019), Rentenversicherung in Zahlen, eigene Darstellung

\subsection{Hinterbliebenenrenten der Gesetzlichen Rentenversicherung}

Die Darstellung der Versichertenrenten hat gezeigt, dass die eigenständig von Frauen erwirtschafteten Renten nach wie vor (deutlich) hinter denen der Männer zurückbleiben. Allerdings können die geringen Renten der Frauen im Haushaltskontext durch die Alterseinkommen ihrer (Ehe-)Männer ausgeglichen werden. Aber das funktioniert nur im Falle von stabilen Verbindungen, in denen beide Partner ein ähnliches Alter erreichen. Der Tod des Ehepartners und vor allem die Scheidung entziehen dieser Konstellation ihre Wirkung. Zwar wird im Fall der Scheidung ein Versorgungsausgleich durchgeführt, bei dem die in der Ehe erworbenen Rentenanwartschaften in der Regel hälftig zwischen den Partnern aufgeteilt werden. Aber dabei wird die häufig asymmetrische Aufgabenverteilung in der Ehe und die daher zu erwartenden Einkommensverluste in der auf die Ehe folgende Erwerbsphase, nicht berücksichtigt (vgl. Klammer 2017, S. 364). Schließlich wirkt sich ein familienbedingter (vorübergehender) Erwerbsausstieg oder eine (geringfügige) Teilzeitbeschäftigung auch dann noch auf den Erwerbs- und Einkommensverlauf aus, wenn den Betroffenen (in 
der Regel Frauen) wieder eine Vollzeittätigkeit möglich ist. Im Ergebnis liegt der sogenannte Gender Lifetime Earnings Gap, das die Lebenseinkommenslücke zwischen den Geschlechtern misst, bei 49,8 Prozent (Boll et al. 2017, S. 64). Das bedeutet, dass Frauen im Durchschnitt über ihren ganzen Erwerbsverlauf hinweg lediglich etwa die Hälfte der männlichen Erwerbseinkommen realisieren (können). Auch das ist ein Erklärungsfaktor für die geschlechtsspezifischen Unterschiede in der Rentenhöhe.

Im Falle einer Ehe bis zum Tod (des Mannes) „erben“ Frauen zwar Hinterbliebenenrenten, aber diese Rentenart begreift die Anwartschaften des Verstorbenen nicht grundsätzlich als gemeinsam erwirtschafteten Anspruch eines Ehepaares, die dann auf den oder die Hinterlasse/n übertragen werden. Hinterbliebenenrenten haben einen anderen sozialrechtlichen Status als eigenständig erworbene Anwartschaften, sie sind rechtlich als eine Art Zwitter „zwischen einer Versicherungsleistung und einer Fürsorgeleistung“ (Klammer 2012, S. 175) einzuordnen. Das bedeutet vor allem, dass sie nicht grundsätzlich darauf abzielen, hinterbliebene Frauen so abzusichern, dass sie ihren Lebensstandard weiter halten können. Es greift vielmehr das Individual- und Nachrangprinzip: Der Lebensunterhalt der/des Hinterbliebenen soll eigentlich eigenständig finanziert werden, lediglich ein fest definierter Freibetrag kann in jedem Fall weitervererbt werden. Kürzungen und die Anrechnung von eigenem Einkommen sind in jedem Fall vorgesehen. So beträgt die große Witwenrente lediglich 55 (in Altfällen 60) Prozent der Vollrente des Verstorbenen. Eigene Einkünfte (nicht nur eigene Versichertenrenten und Arbeitsentgelte, sondern nahezu alle Einkommensarten) oberhalb eines Freibetrags ${ }^{2}$ werden zu 40 Prozent auf die Hinterbliebenenrenten angerechnet. Dadurch kann der Anspruch auf die ausgezahlte Hinterbliebenenversorgung sich mindern oder vollständig ruhen. Das eigene Erwerbseinkommen von Frauen führt beim Tod des Ehemannes beispielsweise tendenziell zu Kürzungen der Hinterbliebenenrente. Im Falle einer neuen Eheschließung entfallen die abgeleiteten Ansprüche (anders als eigenständig erworbene) Anwartschaften nach einer Übergangsfrist außerdem gänzlich ${ }^{3}$. Dadurch entstehen hohe Opportunitätskosten, die einem Großteil der Betroffenen jedoch gar nicht bewusst sein dürften (vgl. Klammer 2012, S. 176).

2 Aktuell 872,52 € West und 841,90 € Ost (zuzüglich etwaiger Aufschläge im Falle von Kindern mit Anspruch auf Waisenrente).

3 Einen Ausnahmefall stellen die im Rahmen des Rentensplittings aufgeteilten Rentenansprüche dar. Bei diesem Verfahren werden die in der Ehezeit erworbenen Anwartschaften zu gleichen Teilen unter den Ehepartnern aufgeteilt. Allerding ist dieses Verfahren nur dann möglich, wenn die Eheleute das Erwerbsleben bereits abge- 
Insgesamt steht die Hinterbliebenenversorgung im Kontrast zu sozialund steuerrechtlichen Regelungen, die nach wie vor starke Anreize dafür setzen, dass in der Familienphase hauptsächlich ein Ehepartner oder eine Ehepartnerin die Erwerbsarbeit übernimmt (siehe unten). Denn einen Rechtsanspruch auf die ungekürzten Anwartschaften hat nur derjenige, der die Anwartschaften auch erworben hat. In seiner Wirkung stellt das „Anrechnungs-Modell“ folglich vor allem Frauen schlechter. Auch wenn die Hinterbliebenenrenten im Leistungsvolumen der gesetzlichen Rentenversicherung (insbesondere für Frauen) nach wie vor eine große Rolle spielen, so sind durch diese Regelungen dennoch Versorgungslücken vorprogrammiert. Trotzdem scheint ein nennenswerter Teil der Frauen auf diese Rentenart zu setzen. Das gilt insbesondere für Westdeutschland. Hier weisen die Witwenrenten im Schnitt sogar ein höheres Niveau auf als die Altersrenten von Frauen. Das zeigt sich auch bei der Schichtung der Renten: Im Jahr 2018 erhielten 23,1 Prozent der Frauen eine eigene Rente mit einem Zahlbetrag von weniger als $300 €$, bei den Witwenrenten waren es 19,2 Prozent. Einen Zahlbetrag von weniger als $600 €$ findet sich bei der Hälfte $(50,6 \%)$ der Frauenaltersrenten, aber "nur" bei $41 \%$ der Witwenrenten. Umgekehrt ist es nur bei höheren Renten, hier sind die Altersrenten ein wenig stärker vertreten: Eine Witwenrente von $1.200 €$ erhalten lediglich 3,1 \% der Witwen, aber 9,6\% der Frauen mit eigenen Anwartschaften.

\subsection{Betriebliche und private Altersvorsorge}

Die besondere Abhängigkeit des Rentensystems von der Konstellation auf dem Arbeitsmarkt gilt nicht nur für die Renteneinkommen aus der gesetzlichen Rentenversicherung, sondern mehr noch für die Einkünfte aus der betrieblichen und privaten Vorsorge. Im Vergleich zur gesetzlichen Rentenversicherung ist der interne Solidarausgleich auf diesen beiden Ebenen zudem schwächer ausgeprägt (betriebliche Altersvorsorge) bzw. gar nicht vorhanden (private Vorsorge), so dass hier die Erwerbsteilhabe der Frauen

schlossen haben. Damit sich das Rentensplitting lohnt, muss außerdem in der Regel eine - möglichst wahrscheinliche - Prognose darüber getroffen werden, welcher Partner zuerst verstirbt. Dementsprechend bietet sich das Verfahren nur für ausgewählte Fälle an. 
vollständig durchschlägt. ${ }^{4}$ Gleichzeitig - und das gilt für alle Rentnerinnen und Rentner in gleicher Weise - sind durch die Reformen der letzten beiden Dekaden systematische Sicherungslücken entstanden, die nur unter optimistischen Annahmen durch eine zusätzlich Vorsorge in den auf Freiwilligkeit beruhenden betrieblichen und privaten Systemen geschlossen werden können.

Aktuell lässt sich die Situation durch die gegenwärtig gezahlten Betriebs- und Privatrenten an die Rentnerinnen und Rentner auf der einen Seite und den Anteil der aktiven Erwerbsbevölkerung, die betriebliche oder private Vorsorgeprodukte besparen, beschreiben. Allerdings ist die Datenlage in der zweiten und dritten Säule wesentlich unübersichtlicher als im Bereich der gesetzlichen Rente. Schließlich gibt es nicht das eine betriebliche oder private Produkt, sondern eine Vielzahl von unterschiedlichen Vorsorgemöglichkeiten und Anbietern. Eine Annäherung bietet die vom Bundesministerium für Arbeit und Soziales im Abstand von vier Jahren herausgegebene Studie „Alterssicherung in Deutschland“ (ASID), in der die Gesamteinkommen im Alter erhoben werden. Außerdem erstellt die Bundesregierung alle vier Jahre den so genannten „Alterssicherungsbericht", der unter anderem ebenfalls die zusätzlichen Altersvorsorge thematisiert.

Die aktuellsten Daten liegen für das Jahr 2015 vor. Sie zeigen, dass unter der älteren Bevölkerung ab 65 Jahren in Westdeutschland etwa $31 \%$ der Männer aber gerade einmal $8 \%$ der Frauen eine Betriebsrente beziehen. Auch die Zahlbeträge liegen weit auseinander: Während sich die durchschnittliche Betriebsrente der westdeutschen Männern auf $606 €$ beläuft, liegt die Durchschnittsbetriebsrente der Frauen lediglich bei $246 €$. Im Osten Deutschlands spielen Betriebsrenten derzeit faktisch keine Rolle. Lediglich $5 \%$ der Männer und nur $1 \%$ der Frauen verfügt überhaupt über Renteneinkünfte aus der zweiten Säule. Auch die Höhe der durchschnittlichen Betriebsrenten fällt mit $210 €$ (Männer) und $115 €$ (Frauen) deutlich geringer aus als im Westen. Darüber hinaus sind unter den jetzigen Rentnerinnen und Rentnern private Renten sowohl in West- als auch in Ostdeutschland kaum verbreitet. Im Westen Deutschlands beziehen lediglich $5 \%$ der Männer ( dem privaten System. In Ostdeutschland sind es nur 3\% der Männer

4 Allerdings sind in der Förderung der Riester-Rente bspw. Kinderzulagen vorhanden. Damit werden bei der Prämienzahlung soziale Gesichtspunkte berücksichtig; s. auch den Beitrag von Bäcker in diesem Band. 
(Ø $150 €)$ und $1 \%$ der Frauen (Ø $172 €)$, die im Rentenalter über eine private Renten- oder Lebensversicherung verfügen (vgl. Bundesministerium für Arbeit und Soziales 2017).

Nun sagt der gegenwärtige Bezug von betrieblichen und privaten Renten nichts darüber aus, wie sich die Renten in diesen Systemen in Zukunft entwickeln werden. Schließlich müssen auch die Reformen und systematische Förderung der zweiten Säule (etwa durch Entgeltumwandlung oder Eichel-Förderung) und der dritten Säule (Riester-Rente) erst ihre Wirkung entfalten. Allerdings lässt sich beobachten, dass die Erwartungen an die betriebliche und private Altersvorsorge bislang unerfüllt geblieben sind. Die Empirie zeigt, dass der Abdeckungsgrad beider Säulen nach wie vor unbefriedigend ist und eine hohe soziale Selektivität aufweist. Die aktuellsten Daten belegen, dass gegenwärtig (2017) etwa 55,6 Prozent der sozialversicherungspflichtig Beschäftigten bAV-Anwartschaften erwerben (Bundesministerium für Arbeit und Soziales 2019b, S. 15). Dabei zeigen sich erhebliche Ungleichheiten: Beschäftigte in großen Betrieben und bestimmten Wirtschaftszweigen (wie die Kredit- und Versicherungswirtschaft, das verarbeitende Gewerbe oder Bergbau/Steine/Erde/Energie) erwerben deutlich häufiger betriebliche Rentenanwartschaften als Personen, die in kleinen Unternehmen oder der Dienstleistungsbranche und dem Gastgewerbe tätig sind (vgl. Baumann/Blank 2016, S. 16-137). Allerdings finden sich genau in den zuletzt genannten Branchen vermehrt Frauen, auch in kleinen Betrieben sind sie überrepräsentiert. Aus diesem Gründen sind Frauen im System der betrieblichen Altersvorsorge auch nach wie vor deutlich schlechter gestellt als Männer.

Darüber hinaus gilt, dass die Verbreitung allein nichts über die Höhe der eingezahlten Beiträge, die zu erwartenden Renten und deren Anpassung im Zeitverlauf aussagt. Wie hoch die zu erwartenden Renten aus der betrieblichen Altersvorsorge sind, ist also völlig unklar. Das trifft in noch stärkerem Maße auf die private Vorsorge zu. Hier ist die Lage noch weitaus unübersichtlicher: Das Bundesministerium für Arbeit und Soziales weist für das Jahr 2018 16,592 Mio. Riester-Verträge aus (Bundesministerium für Arbeit und Soziales 2019a). Das bedeutet aber nicht, dass auch 16,592 Mio. Personen privat vorsorgen, denn die Daten werden nicht personenbezogen erfasst. So zeigen die Daten des Finanzministeriums zur Riester-Förderung, dass im Jahr 2018 lediglich 10,8 Mio. Personen (vorläufige Angabe) eine Förderung erhielten (Bundesministerium der Finanzen 2019). Setzt man diese Daten ins Verhältnis zu den etwa 39 Mio. unmittelbar und mittelbar Förderberechtigten insgesamt, so kann von einer flächendeckenden Verbreitung der privaten Altersvorsorge keine Rede sein. Darüber hinaus schätzt die Bundesanstalt für Finanzdienstleistungsaufsicht nach Angaben 
der Bundesregierung, dass derzeit rund ein Fünftel aller Riester-Verträge ruhend gestellt sind, also gar nicht bespart werden (Deutscher Bundestag 2017).

\section{Frauen und Arbeit}

Die beträchtlichen Unterschiede zwischen den Alterseinkünften von Männern und Frauen basieren auf der geschlechtlichen Arbeitsteilung in Haushalten und der unterschiedlichen Arbeitsmarktsituation von Männern und Frauen. Denn grundsätzlich gilt, dass die individuelle Rentenhöhe eine Art Spiegelbild der vorangegangenen Erwerbsbiografie ist. Im Vergleich zu anderen europäischen Ländern orientiert sich die Rentenberechnung in Deutschland - trotz erheblicher Reformen und leistungsrechtlicher Einschnitte - nach wie vor stark an dem vorherigen Einkommen. Ohne an dieser Stelle in die Details der Rentenberechnung zu gehen, lässt sich festhalten, dass die Höhe der individuellen Renten durch die Anzahl der Erwerbsjahre (Zeitfaktor) und der Höhe des Erwerbseinkommens (Einkommensfaktor; vgl. Klammer 2017, S. 361) bestimmt wird. Es gilt außerdem das Äquivalenz- bzw. Lohnersatzprinzip: Wer lange versicherungspflichtig gearbeitet und eine gute Verdienstposition erreicht hat, wird eine höhere Rente erzielen als Beschäftigte, die nur wenige Beschäftigungsjahre aufweisen und/oder wenig verdient haben. Diese Konstruktionslogik führt vor allem für Frauen zu einer erhöhten Gefahr von niedrigen eigenen Alterseinkünften. Dabei beeinträchtigen Arbeitslosigkeit oder (familienbedingte) Erwerbsunterbrechungen über den Zeitfaktor die Rentenhöhe. Teilzeit, nur marginal versicherte Minijobs, niedrige Frauenlöhne und ausgebliebene Karriereentwicklungen dämpfen die Rentenansprüche über den Einkommensfaktor. Beide Faktoren wirken sich regional unterschiedlich aus: Westdeutschen Rentnerinnen mangelt es im Vergleich zu den Männern sowohl an Erwerbsjahren (Zeitfaktor) als auch Einkommenshöhe (Einkommensfaktor). Ostdeutsche Rentnerinnen hingegen leiden immer noch unter den bereits zu DDR-Zeiten vorhandenen geschlechtsspezifischen Einkommensdifferenzen. Ihnen fehlen zwar nur wenige Beitragsjahre, aber die durchschnittlich niedrigeren Jahreseinkommen führen nach wie vor zu niedrigen Frauenrenten (vgl. Grabka et al. 2017, S. 88; Klammer 2017, S. 361).

Bereits diese hier nur überblicksartige Zusammenfassung verweist darauf, dass es sich bei der Arbeitsmarktsituation von Frauen um eine multiple Problemlage handelt. Es geht um weit mehr als die Vorstellung des klassischen „Drei-Phasen-Models“ von Erwerbstätigkeit, Familienphase 
und beruflichen Wiedereinstieg von Frauen, das in dieser Grundform nicht allein zur Erklärung der weiblichen Erwerbs- und Rentenprobleme ausreicht (vgl. Clemens 1997, S.9). Vielmehr sind Frauen auf dem deutschen Arbeitsmarkt nach wie vor mit einer Reihe von Benachteiligungen konfrontiert, die sich gegenseitig überlagern und ergänzen. $\mathrm{Zu}$ den wesentlichen Aspekten zählen neben der Dauer und dem Umfang der Erwerbstätigkeit auch die Verteilung auf Berufe (horizontale Segregation), der Zugang zu Führungspositionen (vertikale Segregation) und nicht zuletzt die geschlechtsspezifischen Lohnunterschiede. Sie werden im Folgenden überblicksartig nebeneinandergestellt.

\subsection{Arbeitszeit}

In der sozialpolitischen Debatte sind die Arbeitszeitunterschiede zwischen den Geschlechtern ein Dauerthema. Allerdings wird die Diskussion mitunter durch Darstellungen verzerrt, die die Frauenerwerbsbeteiligung nur unvollständig abbilden. Als positive Entwicklungstendenz wird in diesem Zusammenhang häufig die gestiegene Erwerbstätigenquote der Frauen herangezogen. Die Zahl gibt an, welcher Anteil der männlich bzw. weiblichen Bevölkerung zwischen 15-65 Jahren überhaupt einer Erwerbstätigkeit nachgeht. Die Entwicklung ist tatsächlich erfreulich: In Westdeutschland waren im Jahr 1991 lediglich 56,5 \% der Frauen aber 78,4 \% der Männer erwerbstätig. Knapp dreißig Jahre später hat sich diese Bild stark gewandelt: Im Jahr 2018 gingen 71,6\% der Frauen und 80\% der Männer einer Erwerbstätigkeit nach. Auch wenn die Quoten im Zeitverlauf beträchtlichen Schwankungen unterlagen, so hat sich die reine Erwerbsteilhabe zwischen den Geschlechtern in Westdeutschland stark angenähert. Im Osten Deutschlands waren die Unterschiede zwischen Männern und Frauen bereits im Jahr 1991 nicht derart deutlich ausgeprägt. 78,5\% der Männer und $66,8 \%$ der Frauen waren zu dieser Zeit erwerbstätig. Aber auch hier ist es zu einer deutlichen Angleichung gekommen. Im Jahr 2018 lag die Erwerbstätigenquote der Männer bei 78,0 \%, während die der Frauen 73,9\% betrug (Datenquelle: Statistisches Bundesamt 2019, Mikrozensus, eigene Berechnungen).

Allerdings verharrt das weibliche Arbeitsvolumen im Zeitverlauf trotz der dynamisch wachsenden Frauenerwerbstätigenquote insgesamt auf einem annährend konstanten Niveau. Das ist darauf zurückzuführen, dass die Zunahme der Erwerbsbeteiligung der Frauen vor allem auf der wachsenden Verbreitung von Teilzeitbeschäftigungsverhältnissen beruht. Im Jahr 2018 war fast die Hälfte (47,0 \%) aller abhängig beschäftigten Frauen 
in Teilzeit tätig. Die Teilzeitquote der Männer lag zum gleichen Zeitpunkt lediglich bei $11,2 \%$. Dementsprechend zeigt sich auch über alle Lebensphasen hinweg ein deutlicher Unterschied zwischen dem durchschnittlichen Erwerbsumfang (in Stunden) von Männern und Frauen. Durchschnittlich liegt die wöchentliche Arbeitszeit der Frauen mit 29,8 Stunden vor allem in Westdeutschland deutlich unterhalb des Vollzeitstandards. Im Zeitverlauf wird außerdem sichtbar, dass die Arbeitsstunden pro Frau von 1991 (33,2 Stunden) bis 2006 (29,3 Stunden) kontinuierlich zurückgegangen sind und seitdem konstant bleiben (2018: 29,8 Stunden). Auch im Osten Deutschlands sind die normalerweise geleisteten Wochenarbeitsstunden von Frauen im Zeitverlauf gesunken, von 38,3 Stunden im Jahr 1991 auf 33,6 Stunden im Jahr 2018.

Die Durchschnittswerte variieren je nach Altersgruppe noch einmal beträchtlich. Am größten fallen die geschlechtsspezifischen Arbeitszeitunterschiede bei Familien mit kleinen Kindern aus. Darin kommt das Problem zum Ausdruck, Berufstätigkeit und Familie miteinander zu vereinbaren. Die nach wie vor fehlende Kinderbetreuungsinfrastruktur mag hier als Stichwort reichen. Offensichtlich muss die "rush hour des Lebens [...] von den Frauen durch eine geringere Erwerbsbeteiligung entschärft" werden (Klammer 2009, S. 58). Das schlägt sich auch in einer geschlechtsspezifischen Zeitverwendung insgesamt nieder. Frauen schultern im Lebensverlauf nach wie vor einen Großteil der unbezahlten Haus- und generativen Sorgearbeit. Der Gender Care Gap ${ }^{5}$ betrug im Jahr 2012/13 52,4 Prozent (Sachverständigenkommission zum Zweiten Gleichstellungsbericht der Bundesregierung 2017, S.38). Das bedeutet, dass Frauen täglich um die Hälfte mehr (etwa 1,5 Std pro Tag) unbezahlte Sorgearbeit leisten als Männer. Die lapidare Formulierung, dass Frauen auch heute noch „weniger arbeiten" geht daher fehl. Aber ihre Teilhabe an der bezahlten Lohnarbeit steht nach wie vor hinter den Männern zurück, und das führt unter den gegebenen rentenrechtlichen Bedingungen zu einer Schlechterstellung im Altersvorsorgesystem.

5 Der Gender Care Gap wurde erstmals durch die Sachverständigenkommission zum zweiten Gleichstellungsbericht errechnet. Es erfasst den relativen Unterschied in der täglich für Care-Arbeit verwendeten Zeit zwischen Männern und Frauen. Es gibt an, um wieviel Prozent die Zeit, die Frauen im Durchschnitt pro Tag für CareArbeit aufwenden, die durchschnittliche Dauer der täglichen Care-Arbeit von Männern übersteigt. Die Kommission empfiehlt, den Indikator zukünftig periodisch zu berechnen, um die unterschiedliche Zeitverwendung zwischen den Geschlechtern sichtbar zu machen (vgl. Sachverständigenkommission zum Zweiten Gleichstellungsbericht der Bundesregierung 2017, S. 38). 


\subsection{Horizontale und vertikale Segregation}

Ein weiteres Kennzeichen der Frauenerwerbsbeteiligung ist die nach wie vor starke horizontale und vertikale Segregation des deutschen Arbeitsmarktes. Das Schlagwort der horizontalen Segregation bezieht sich darauf, dass sich die sozialversicherungspflichtig beschäftigten Männer und Frauen nicht in gleicher Weise auf die verschiedenen Berufe verteilen. Dabei gelten Berufssegmente mit einem Anteil von mehr als 70\% des jeweiligen Berufes als Frauen bzw. Männerdomänen (Hobler et al. 2019). Frauendominierte Berufe finden sich vor allem im Bereich der personenbezogenen Dienstleistungen wie Pflege, Erziehung und Reinigung sowie im Bereich einfacher Bürotätigkeiten. Technische und verarbeitende Berufe hingegen werden von Männern dominiert. Eine relative Gleichverteilung findet sich lediglich im Bereich der Lebensmittel- und Gastgewerbeberufe, Handelsberufe sowie unternehmensbezogenen Dienstleistungen (Hausmann/Kleinert 2014, S. 1).

Grundsätzlich wäre diese Schieflage völlig unproblematisch, ginge die unterschiedliche Verteilung der Geschlechter auf die Berufe nicht mit einer systematisch schlechteren Bewertung der frauendominierten Felder einher. Das bezieht sich nicht nur auf die eklatanten Lohnunterschiede (siehe 3.3), sondern beginnt bereits im Bereich der beruflichen Ausbildung. Während typische Männerberufe in der Regel betrieblich (und entlohnt) ausgebildet werden, geht eine Vielzahl typischer Frauenberufe (vor allem im sozialen Bereich) mit einer schulischen und damit auch unbezahlten Ausbildung einher, die durch ein so genanntes Anerkennungsjahr abgeschlossen wird. Die Schlechterstellung weiblich dominierter Berufe wird auf diese Weise schon vor dem eigentlichen Eintritt in den Arbeitsmarkt institutionell verfestigt.

Neben der Unterscheidung in Männer- und Frauenberufe finden sich Frauen auf dem Arbeitsmarkt auch häufiger in rangniedrigeren Positionen oder Statusgruppen. Im Vergleich zu ihrem Beschäftigungsanteil erreichen sie Führungspositionen deutlich schlechter als ihre männlichen Kollegen (vertikale Segregation). Das gilt für vollzeitbeschäftigte und in noch stärkerem Maße für teilzeitbeschäftigte Frauen (Hobler et al. 2018). Allerdings erklärt der Beschäftigungsumfang allein die vertikale Segregation nicht. Unter Schlagworten wie "gläserne Decke“, „statistische Diskriminierung“, „Old-Boys-Networks“ oder dem „Prinzip der Selbstähnlichkeit“ herrscht in der wissenschaftlichen Debatte weitestgehend Einigkeit darüber, dass es nach wie vor eine Reihe von betrieblichen Gründen gibt, die die vertikale Segregation erklären. Ohne an dieser Stelle in die Details der mitunter hitzig geführten Debatte zu gehen lässt sich festhalten, dass sich stereotype 
Rollenerwartungen sowohl von Einstellenden als auch bei Vorgesetzten hartnäckig halten. In ihrer Sichtweise gelten Frauen häufig als nicht kalkulierbares ökonomisches Risiko, weil jede Frau eine potenzielle Mutter ist, die entweder phasenweise ganz ausfällt oder aufgrund der unterstellten Doppelbelastung von Berufstätigkeit und Hausarbeit oder Berufstätigkeit und Familie in ihrem Leistungsvermögen begrenzt ist. Für die Renteneinkommen von Frauen sind verhinderte Karrieresprünge deswegen problematisch, weil die fehlenden Lohnsteigerungen zu Beitrags- und damit auch Leistungseinbußen in der Rente führen.

\subsection{Lohnunterschiede}

Ein drittes Kennzeichen der Frauenerwerbsbeteiligung sind die geschlechtsspezifischen Lohnunterschiede. Auch sie erweisen sich im Zeitverlauf als ausgesprochen robust und werden statistisch häufig durch die Maßzahl des Gender Pay Gaps ${ }^{6}$ ausgewiesen. Es liegt in den letzten zehn Jahren im Durchschnitt relativ konstant bei einem Wert von etwa $22 \%$ (unbereinigt) bzw. $7 \%$ (bereinigt).

Das Statistische Bundesamt berechnet den Gender Pay Gap auf Basis der vierteljährlichen Verdienststrukturerhebung. Als Bemessungsgrundlage dient der durchschnittliche Bruttostundenverdienst abhängig beschäftigter Männer und Frauen. In der so genannten bereinigten Variante wird der Teil der Verdienstunterschiede herausgerechnet, der auf Unterschieden zwischen den Geschlechtern wie der unterschiedlichen Verteilung auf Berufe, dem Beschäftigungsumfang, dem Bildungsstand oder der Berufserfahrung beruht. Häufig folgt daraufhin eine Interpretation, die nur den bereinigten Gender Pay Gap als gleichstellungspolitisch relevante Größe betrachtet. Aufgrund der geringen Höhe des bereinigten Gender Pay Gaps wird mitunter auch geschlussfolgert, dass gar kein wesentlicher sozialpolitischer Handlungsbedarf besteht. Dass durch die Art der Bereinigung gerade die horizontale Arbeitsmarktsegregation und mit ihr die Abwertung,weiblicher Berufe' aus der Statistik herausgerechnet werden, wird nicht weiter besprochen. Das ist problematisch, denn Frauen arbeiten in der Regel in schlechter bezahlten Berufen und Branchen. Genauso gilt andersrum:

6 Der Gender Pay Gap misst den prozentualen Anteil, den Frauen im Durchschnitt pro Arbeitsstunde weniger verdienen als Männer. Als Bemessungsgrundlage dient der durchschnittliche Bruttostundenverdienst abhängig beschäftigter Männer und Frauen (vgl. Hobler/Pfahl 2017; Klenner et al. 2016). 
Frauenberufe oder Frauenbranchen werden in der Regel schlechter bezahlt. Allerdings handelt es sich dabei nicht - wie in der neoklassischen Sichtweise häufig unterstellt - um das legitime Ergebnis eines perfekt funktionierenden Marktes, der einen "gerechten“"Wert von Arbeit auf der Basis von neutralen, fair austarierten Angebots- und Nachfrageüberlegungen oder Produktivitätsunterschieden bestimmt. Im Gegenteil: Die Bewertung von Arbeit wird nicht nur durch rechtliche Eingriffe, sondern auch durch die industriellen Beziehungen entscheidend mitbestimmt. Löhne werden häufig in Tarifverträgen oder betrieblichen Vereinbarungen verhandelt und schließlich vereinbart. Dabei müssen Kriterien angelegt werden, wie die einzelnen Tätigkeiten bewertet und bezahlt werden. Wenn in diesem Zuge vor allem als typisch männlich geltende Fertigkeiten (wie bspw. körperliche Kraft) berücksichtigt, aber die als typisch weiblich geltenden Fähigkeiten (wie bspw. Sozialkompetenz) nicht berücksichtigt werden, ergibt sich schon allein durch die Arbeitsbewertungsverfahren eine Schieflage, die statistisch nicht gesehen oder sogar zusätzlich noch „herausgerechnet“ wird (Schmitz 2018, S. 3). Um das tatsächliche Ausmaß der Lohnungleichheit exakter zu beziffern ist es daher notwendig, auch einen Blick auf die Arbeitsbewertungsverfahren zu werfen (vgl. Lillemeier 2016, S. 23). Der Gender Pay Gap im Allgemeinen und die bereinigte Variante im Besonderen sind daher mit Vorsicht zu interpretieren.

Die Auswirkungen der geschlechtsspezifischen Lohnunterschiede auf die spätere Rentenhöhe ist gravierend. Auch bei grundsätzlich gleichen Erwerbsumfang, wohlmöglich sogar im gleichen Beruf, fallen die Beiträge der Frauen zur gesetzlichen Rente und damit auch die zu erwartende Leistungshöhe der Rente deutlich niedriger aus, als bei den männlichen Kollegen. Darüber hinaus ist auch zu unterstellen, dass niedrigere Löhne - ganz allgemein gesprochen - die Sparbereitschaft und Sparfähigkeit in der zweiten und dritten Säule negativ beeinflussen.

\section{Diskussion: Ursachen und Perspektiven}

Ein Blick auf die Alterseinkünfte von Frauen wie auch auf ihre Arbeitsmarktbilanz zeigt, dass Frauen nach wie vor deutlich schlechter gestellt sind als Männer. Denn auch wenn die Frauenerwerbsbeteiligung im Zeitverlauf gestiegen ist, reicht der Umfang des weiblichen Arbeitsvolumens in Verbindung mit Segregation und Lohnunterschieden unter den gegebenen rentenrechtlichen Rahmenbedingungen häufig nicht aus, um eigen- 
ständig eine ausreichende Altersvorsorge betreiben zu können. Das liegt vor allem daran, dass das deutsche Alterssicherungssystem in einem so engen Zusammenhang mit dem Erwerbseinkommen steht.

Die dahinterliegende Idealvorstellung eines Vollzeitbeschäftigungsverhältnisses über das ganze Arbeitsleben hinweg ist aber in der Regel nur auf der Grundlage einer Arbeitsteilung möglich, in der die im Privatbereich anfallende Arbeit ausgelagert wird. Im Zeitverlauf hat sich eine geschlechtsspezifische Trennung entwickelt und verfestigt, nach der Männer den Erwerb des Lebensunterhalts gewährleisten und Frauen die Reproduktionsarbeit im privaten Haushalt übernehmen (vgl. Bäcker et al. 2010a, S. 391). Für eine industrialisierte Arbeitswelt galt diese Form der Arbeitsteilung über lange Zeit als hochgradig funktional. Sie führte aber auch dazu, dass die vor allem für die Haus- und Familienarbeit zuständigen Frauen einen deutlich weniger starken faktischen und normativen Bezug zum Erwerbssystem hatten bzw. haben. Die geschlechtsspezifischen Differenzen sind äußerst robust: Selbst wenn Frauen im gleichen Umfang erwerbstätig sind, übernehmen sie häufig dennoch deutlich mehr Sorge- und Hausarbeit als ihre männlichen Partner (vgl. Hobler et al 2017, S. 6ff). Das gilt auch dann, wenn Frauen mehr verdienen (vgl. Bundesministerium für Familie, Senioren, Frauen und Jugend 2017, S. 99). Von einer Aufhebung der geschlechtsspezifischen Arbeitsteilung kann also nach wie vor nicht die Rede sein.

Allerdings sind es nicht nur normative Vorstellungen, die das Verhalten der Geschlechter auf dem Arbeitsmarkt prägen. Denn die unterschiedlichen Lebenslauf- und Erwerbsmuster von Frauen und Männern werden auch stark durch handfeste institutionellen Rahmenbedingungen beeinflusst. Die ungleiche Arbeitsmarktintegration der Geschlechter ist auch ein Ergebnis der geschlechtsspezifisch kontrastierenden Anreizstrukturen und institutionell verankerten Wirkungsmechanismen. Im Steuer- und Sozialrecht findet sich dafür eine Fülle von Beispielen: Neben dem Ehegattensplitting, der Steuerklassenkombination III/V oder der kostenfreien Mitversicherung der nicht oder nur marginal erwerbstätigen Frau in der Krankenversicherung ist auch die Hinterbliebenenversorgung in der gesetzlichen Rente zu nennen.

Um die materielle Situation von Frauen im Rentenalter nachhaltig zu verbessern, sind vor diesem Hintergrund zwei Einflussbereiche zu nennen, auf denen staatliche Eingriffe und rechtliche Reformen notwendig erscheinen: Im Bereich des Arbeitsmarkts sind erstens Regelungen von Nöten, die sowohl die Erwerbsintegration als auch die Stellung von Frauen in der Arbeitswelt grundlegend verbessern. Hierzu müssen die zuvor genannten $\mathrm{Ne}$ gativanreize, die der Erwerbsbeteiligung von Frauen entgegenstehen, zu- 
nächst behoben werden. Zu nennen sind insbesondere die Abschaffung der steuerfreien Mini-Jobs sowie die kostenfreie Mitversicherung in der Krankenversicherung. Auch der flächendeckende Ausbau einer Kinderbetreuungsinfrastruktur dürfte das Erwerbsverhalten der Frauen nachhaltig stärken. Darüber hinaus ist es unabdingbar, das berufliche Fortkommen von Frauen zu fördern und die Lohnunterschiede abzubauen. Neben einer echten Frauenquote für betriebliche Führungspositionen ist es auch denkbar, die Betriebe auf die Analyse ihrer eigenen Arbeitsbewertungsverfahren zu verpflichten und darüber regelmäßig öffentlich zu berichten. Allein diese Transparenz dürfte den Druck erhöhen, alle Aufgaben und Belastungen bei der Lohnfindung zu berücksichtigen. Hilft das nichts so könnte die gleiche Bezahlung von Männern und Frauen auch nach isländischem Vorbild gesetzlich vorgeschrieben und Verstöße mit Bußgeldern geahndet werden.

Als zweiter Einflussbereich sind die rentenrechtlichen Rahmenbedingungen zu nennen. ${ }^{7}$ Um die Situation von Frauen zu verbessern, wird häufig eine Aufwertung von Niedriglöhnen oder eine bessere Anrechnung von Zeiten, in denen Care-Tätigkeiten (Pflege und Kindererziehung) verrichtet wurden, gefordert. Das ist gut und richtig. Allerdings würden diese Maßnahme das grundsätzliche Dilemma von unterstelltem Vollzeitstandard und geschlechtsspezifischer Arbeitsteilung zwar entschärfen, aber nicht lösen. Nachhaltiger wäre es daher, hier zumindest für sozialrechtliche Konsequenz zu sorgen. Solange es staatliche Anreize gibt, die es attraktiv machen, dass (Ehe-)Paare ihren Lebensunterhalt im aktiven Erwerbsalter phasenweise nur oder vor allem durch ein (in der Regel männliches)

7 All diese Regelungen beziehen sich allein auf die Gesetzliche Rentenversicherung. Das liegt daran, dass die Rentenvorsorge von Frauen nur in diesem Bereich gezielt und nachhaltig verbessert werden kann. In der zweiten und dritten Säule ist es wesentlich schwieriger, die Situation von Frauen zu stärken. Das größte Problem für die Versicherten im Allgemeinen und Frauen im Besonderen besteht in der lückenhaften Verbreitung von betrieblichen und privaten Anwartschaften, die sich auf die Freiwilligkeit der beiden Systeme zurückführen lässt. Die Einführung einer Vorsorgepflicht auch im betrieblichen und mehr noch privaten System ist aber mit Problemen behaftet. Denn das würde die Frage aufwerfen, ob man die Beschäftigten zur Vorsorge auf dem privaten, in der Regel auf Gewinn ausgerichteten Versicherungsmarkt, zwingen kann. Um dieses Problem zu umgehen, wird in diesem Zusammenhang häufig die Einführung eines staatlich organisierten Standard- oder Basisproduktes gefordert, das einfach, transparent und kostengünstig ist. Dazu muss die Durchführung bei einer staatlichen Institution liegen - aber wie ließe sich dann noch die Leistungsabsenkung in der gesetzlichen Rentenversicherung rechtfertigen?. 
Erwerbseinkommen bestreiten, kann es nur folgerichtig sein, dass dieses Einkommen pflichtmäßig auch zur Altersvorsorge für beide Lebenspartner herangezogen wird und in zwei eigenständigen Renten mündet. Dazu müsste die Verbeitragung von Einkommen vom Erwerbsstatus abgekoppelt und eine allgemeine Beitragspflicht eingeführt werden. Als Varianten sind eine Mindestversicherungspflicht unabhängig vom Erwerbsstatus oder ein dauerhaftes Rentenanwartschaftssplitting denkbar, bei dem die in der Familienphase erworbenen Ansprüche direkt auf die Rentenkonten der Männer und Frauen aufgeteilt werden. Auf diese Weise würde sich zumindest der Charakter der durch die Partner erwirtschafteten Rentenanwartschaften verändern. Die Schieflage zwischen weiblicher Lebensleistung und realisiertem Alterseinkommen ließe sich so zumindest in Teilen begradigen.

\section{Literatur}

Baumann, H./Blank, F. (2016): Die betriebliche Altersvorsorge. Verbreitung und Finanzierung - Ergebnisse der WSI-Betriebsrätebefragung 2015, WSI Report Nr. 30, Düsseldorf

Boll, C./Jahn, M./Lagermann, A./Puckelwald, J. (2017): Dauerhaft ungleich - berufsspezifische Lebenserwerbseinkommen von Frauen und Männern in Deutschland, HWWI Policy Paper Nr. 98, Hamburg

Bundesministerium für Arbeit und Soziales (2016): Ergänzender Bericht der Bundesregierung zum Rentenversicherungsbericht 2016 gemäß $\$ 154$ Abs. 2 SGB VI. Alterssicherungsbericht 2016, Berlin

Bundesministerium für Arbeit und Soziales (2017): Alterssicherung in Deutschland 2015 (ASID 2015), Endbericht und Tabellenanhang, https://www.bundesregieru ng.de/breg-de/service/publikationen/endbericht-alterssicherung-in-deutschland-2 015-asid-2015--729196 (abgerufen im Januar 2019)

Bundesministerium für Arbeit und Soziales (2019a): Entwicklung der Riester-Verträge, https://www.bmas.de/SharedDocs/Downloads/DE/Thema-Rente/entwickl ung-riester-vertraege.pdf;jsessionid=9BB64C0A2324F736491A7DAB33D1CDA3? blob=publicationFile\&v=16 (abgerufen im Januar 2020)

Bundesministerium für Arbeit und Soziales (2019b): Trägerbericht zur Verbreitung der betrieblichen Altersvorsorge. Forschungsbericht 523, Berlin

Bundesministerium der Finanzen (2019): Statistische Auswertungen zur RiesterFörderung, https://www.bundesfinanzministerium.de/Content/DE/Standardarti kel/Themen/Steuern/Weitere_Steuerthemen/Altersvorsorge/2019-11-15-Statistisc he-Auswertungen-Riester-Foerderung-bis-2018.html (abgerufen im Januar 2020)

Clemens, W. (1997): Frauen zwischen Arbeit und Rente. Lebenslagen in später Erwerbstätigkeit und frühem Ruhestand, Wiesbaden 
Deutsche Rentenversicherung Bund (2019), Rentenversicherung in Zahlen 2019, Berlin

Deutscher Bundestag (2017): Fragestunde der 236. Sitzung des Deutschen Bundestages am Mittwoch, dem 31.05.2017, BT-Drucksache 18/12501, Plenarprotokoll 18/236, Berlin

Flory, J. (2011): Gender Pension Gap. Entwicklung eines Indikators für faire Einkommensperspektiven von Frauen und Männern, https://www.bmfsfj.de/blob/9 3950/422daf61f3dd6d0b08b06dd44d2a 7fb7/gender-pension-gap-data.pdf (abgerufen im Januar 2020)

Grabka, M. M./Jotzo, B./Rasner A./Westermeier C. (2017): Der Gender Pension Gap verstärkt die Einkommensungleichheit von Männern und Frauen im Rentenalter, in: DIW Wochenbericht 5/2017, S. 87-96

Hammerschmid, A./Rowold, C. (2019): Gender Pension Gaps in Europa hängen eindeutiger mit Arbeitsmärkten als mit Rentensystemen zusammen, in: DIW Wochenbericht 25/2019, S. 439-447

Hausmann, A.-C./Kleinert, C. (2014): Berufliche Segregation auf dem Arbeitsmarkt. Männer- und Frauendomänen kaum verändert, IAB-Kurzbericht 9/2014

Hobler, D./Pfahl, S./Horvath, S. (2018): Vertikale Segregation des Arbeitsmarktes 2016, in: GenderDatenPortal des WSI, https://www.boeckler.de/53495.htm (abgerufen im Januar 2020)

Hobler, D./Pfahl, S./Spitznagel, J. (2019): Horizontale Segregation des Arbeitsmarkets 2017, in: GenderDatenPortal des WSI, https://www.boeckler.de/53494.htm (abgerufen im Januar 2020)

Klammer, U. (2012): Hochgebildet - ausgebremst - (re)aktiviert - alimentiert: Frauenerwerbsverläufe in Deutschland, in: Bispinck, R./Bosch, G./Hofemann, K. (Hrsg): Sozialpolitik und Sozialstaat. Wiesbaden, S. 163-188

Klammer, U. (2017): Alterssicherung von Frauen revisted - aktuelle Entwicklungen und zukünftige Perspektiven, in: Sozialer Fortschritt 66 (5), S. 359-375

Sachverständigenkommission zum Zweiten Gleichstellungsbericht der Bundesregierung (2017): Erwerbs- und Sorgearbeit gemeinsam neu gestalten. Gutachten für den Zweiten Gleichstellungsbericht der Bundesregierung, Berlin

Schmitz, J. (2018): Lohnungleichheit: Mit den Belastungen steigt die Bezahlung vor allem bei Männern, sozialpolitik-aktuell.de, Thema des Monats 10/2018 http://www.sozialpolitik-aktuell.de/tl_files/sozialpolitik-aktuell/_Politikfelder/Ei nkommen-Armut/Datensammlung/PDF-Dateien/abbIII2c_Thema_Monat_10_2 018.pdf (abgerufen am Januar 2020)

Sopp, P./Wagner, A. (2011): Gender Pension Gap bei eigenen Alterssicherungsleistungen 1992-2015, in: GenderDatenPortal des WSI, https://www.boeckler.de/11 2210.htm (abgerufen im Januar 2020)

Statistisches Bundesamt (2019): Erwerbstätigenquoten von Männern und Frauen im Zeitverlauf nach Geschlecht und West- und Ostdeutschland. Arbeitstabelle, eigene Berechnungen 